\title{
Prevalence of Germline Mutations in Patients with Pheochromocytoma or Abdominal Paraganglioma and Sporadic Presentation: A Population-Based Study in Western Sweden
}

\author{
Andreas Muth · Frida Abel • Svante Jansson • \\ Ola Nilsson · Håkan Ahlman • Bo Wängberg
}

Published online: 20 January 2012

(C) The Author(s) 2012. This article is published with open access at Springerlink.com

\begin{abstract}
Background Germline mutations in the susceptibility genes RET, $S D H B, S D H D$, and $V H L$ have been reported in 7.5-24\% of patients with pheochromocytoma (Pheo) or paraganglioma (PGL) and sporadic presentation. The purpose of the present study was to establish population-based data on the frequency of germline mutations in patients with apparently sporadic Pheo or abdominal PGL in Western Sweden.

Methods From the Swedish National Cancer Registry, all patients with Pheo or PGL in Western Sweden (population 1.72 million) registered between 1958 and 2009 were identified $(n=256)$. Patients were characterized using register data, hospital records, and clinical interviews. All living patients with Pheo or abdominal PGL and sporadic presentation ( $n=81)$ were invited to genetic screening; 71 patients accepted. Germline mutations were investigated by using direct sequencing for point mutations in RET, $S D H B$, $S D H D$, and $V H L$, and multiplex ligation-dependent probe amplification for gross deletions in $S D H B, S D H C, S D H D$,
\end{abstract}

Preliminary reports of this work have been presented at the 2nd International Symposium on Pheochromocytoma, 2008, Cambridge, UK, and at the MEN2010 meeting, September 2010, Gubbio, Italy.

A. Muth $(\square) \cdot$ S. Jansson · H. Ahlman · B. Wängberg

Department of Surgery, Sahlgrenska University Hospital,

41345 Gothenburg, Sweden

e-mail: andreas.muth@vgregion.se

F. Abel

Genomics Core Facility and Lundberg Laboratory for Cancer

Research, Sahlgrenska Academy, Gothenburg, Sweden

O. Nilsson

Department of Pathology, Sahlgrenska University Hospital,

Gothenburg, Sweden and VHL. Plasma or urinary metanephrines and/or urinary catecholamines were used for biochemical follow-up.

Results The prevalence of germline mutations was 5.6\%. Mutations were only seen in $R E T(n=1)$ and $S D H B$ $(n=3)$. Notably, in the patients with $S D H B$ mutations, no malignant phenotype was observed during a mean followup of 23.3 years.

Conclusions The frequency of germline mutations in patients with apparently sporadic Pheo and abdominal PGL in Western Sweden was lower than in previous studies. Variations in reported frequencies of germline mutations in patients with clinically sporadic Pheo/PGL may reflect geographical differences or patient selection.

\section{Introduction}

The list of known genes causing hereditary pheochromocytoma (Pheo) or paraganglioma (PGL) is expanding and includes $N F 1$ in neurofibromatosis type 1 (NF1), RET in multiple endocrine neoplasia syndrome type 2 (MEN2), VHL in Von Hippel-Lindau syndrome (VHL), succinate dehydrogenase subunit genes $S D H B, S D H C$, and $S D H D$ and the succinate dehydrogenase complex assembly factor 2 gene $S D H A F 2$ in familial paraganglioma syndromes PGL4, PGL3, PGL1, and PGL2, respectively. Recently mutations in PHD2 [1], SDHA [2], TMEM127 [3], Kif- $1 B \beta$ [4], and $M A X$ [5] have been described in association with Pheo/PGL. Since Neumann et al. [6] in 2002 reported 24\% germline mutations in $S D H B, S D H D, R E T$, and $V H L$ in patients with clinically nonsyndromic Pheo/PGL, genetic screening in these patients has attracted considerable interest. An algorithm for testing based on clinical risk factors (family history or age $<35$ years, extra-adrenal, bilateral or malignant tumours) was published in 2006 [7], 
and several modifications have already been suggested [8-10]. In cohorts with Pheo/PGL and sporadic presentation published after 2002, the frequency of germline mutations have ranged from 7.5 to $19.1 \%$ [8-12]. The wide variation may represent geographical differences and/or patient selection, and population-based studies are needed.

To establish population-based data on the frequency of germline mutations in patients with apparently sporadic Pheo or abdominal PGL in Western Sweden, we have offered genetic screening to all living patients from our region registered in the National Cancer Registry (NCR) from 1958 to 2009.

\section{Patients and methods}

\section{Setting}

The western healthcare region in Sweden comprises the region of Västra Götaland and the municipalities Varberg, Falkenberg, and Kungsbacka, with a total population (June 2008) of 1.72 million inhabitants.

\section{Data sources}

\section{National Cancer Registry}

Mandatory reporting of Pheo and PGL to the NCR started in 1958. The reporting frequency is high; approximately $96 \%$ of cases were reported to the NCR according to a recent survey [13]. From the NCR, all patients in the western healthcare region with a diagnosis of Pheo or PGL were identified. Search terms were: ICD-7 localization codes 195.0 (adrenal medulla) and 195.7 (paraganglia); morphology codes 441 (benign Pheo/PGL) and 446 (malignant Pheo/PGL or malignant neuroendocrine tumor). Only patients with histopathologically verified disease were included.

\section{Hospital records and clinical interviews}

From register data, hospital records and clinical interviews information on diagnosis, age at diagnosis, presentation (sporadic or hereditary/syndromic), tumor location and secretory profile, and metastatic or recurrent disease was collected. Sporadic presentation was defined as a negative family history and absence of syndromic lesions associated with MEN2, NF1, or VHL at diagnosis. Malignancy was defined as presence of metastases at the time of diagnosis or during follow-up. To distinguish metastatic from multifocal disease, a diagnosis of metastatic disease required that chromaffin tissue was present at a site where chromaffin tissue is not otherwise found [14]. Because criteria for malignancy in Pheo and PGL have changed over time, histopathological reports were reviewed and in selected cases specimens were reanalyzed to update diagnoses to the current definitions. Plasma or urinary metanephrines and/or urinary catecholamines were used for biochemical follow-up.

\section{Eligibility criteria}

All patients with Pheo or abdominal PGL and sporadic presentation were invited to genetic screening, which started in spring 2006. All participating patients gave oral/ written consent. The study was approved by the Regional Ethical Review Board in Gothenburg (registration number 652-06).

\section{Genetic screening}

Based on the presumed frequency of findings stepwise genetic testing was performed in the following order: (1) Sanger sequencing for point mutations in $S D H B$ (exon 1-8), SDHD (exon 1-4) and $V H L$ (exon 1-3); (2) In patients with negative sequencing results in $S D H B, S D H D$, and $V H L$ sequencing of RET (exons 10,11, 14, and 16); (3) In patients negative after testing for RET-mutation the presence of deletions in $S D H B, S D H C, S D H D$, and $V H L$ was investigated by using multiplex ligation-dependent probe amplification (MLPA).

\section{DNA sequencing}

DNA was enriched from blood by the DNeasy kit from Qiagen (Qiagen, Hilden, Germany) using the Hamilton ML-Star pipetting robot (Hamilton, www.hamiltonrobotics. $\operatorname{com} /$ ).

The primers, covering coding regions in RET gene (Accession number: NM_020975) exon 10,11, 14, and 16 and all coding regions of the $V H L$ (Accession number: L15409), SDHB (Accession number: NM_003000), and SDHD (Accession number: NM_003002) genes were designed using ExonPrimer (http://ihg.gsf.de/ihg/Exon Primer.html) or Primer Express ${ }^{\circledR}$ Software v2.0 (Applied Biosystems), and PCR reactions were set up using the automated workstation Biomek ${ }^{\circledR}$ FX (Beckman Coulter, www.beckmancoulter.com) and performed in 10- $\mu$ l reactions according to standard procedures. PCR products for $V H L, S D H B$, and $S D H D$ were cleaned using magnetic beads (AMPure, Agencourt, Bioscience Corporation, Beverly, MA) and for RET using the Qiagen MinElute PCR Purification kit (Qiagen), and sequenced using BigDye ${ }^{\circledR}$ Terminator v 3.1 Cycle Sequence Kit (Applied Biosystems) in $10-\mu l$ reactions according to manufacturer's protocol. The sequence PCR thermal profile for GC-rich 
fragments (e.g., $V H L$ exon 1) were modified to longer denaturation steps and increased number of cycles (i.e., 50 cycles). The sequence-PCR products were cleaned using magnetic beads (CleanSeq, Agencourt) and separated by gel electrophoresis on a 3730 DNA Analyzer (Applied Biosystems). Results were analysed using Sequencing Analysis v. 5.2 and SeqScape v.2.5 (Applied Biosystems).

$M L P A-S D H B, S D H C, S D H D$, and $V H L$

DNA enriched from blood (cf. DNA sequencing- $S D H B$, $S D H D$, and $V H L$ section) was analyzed for exon deletions by MLPA for $S D H B, S D H C$, and $S D H D$ (SALSA P226, MRC Holland, Amsterdam, The Netherlands) and VHL (SALSA P016-B2) and run according to the manufacturer's recommendations. Results were analyzed using the
GeneMapper v.3.7 software (Applied Biosystems), and normalization was performed in $\mathrm{R}$ 2.9.2. For each case, the MLPA peak heights and peak areas were compared to three independent controls from Western Sweden.

\section{Results}

From 1958 to 2009, 256 patients with Pheo or PGL were registered in the NCR (Fig. 1). Of these, 127 had Pheo or abdominal PGL with sporadic presentation (mean age 52.5 years; $54 \%$ women). Twenty-four patients had hereditary or syndromic presentation (MEN2 $n=13$, NF1 $n=9$, VHL $n=1$, Carney syndrome $n=1$ ), 20 had extra-abdominal paragangliomas, and 1 patient with primary hyperparathyroidism was misclassified in the
Fig. 1 Patients in Western Sweden with pheochromocytoma or paraganglioma 1958-2009. ${ }^{\mathrm{a}}$ Mean age at diagnosis. ${ }^{\mathrm{b}}$ The son of one of the patients had an $S D H B$ mutation (c. $418 \mathrm{G}>\mathrm{T}$ ) but no evidence of disease; the mutation status of the deceased father is unknown

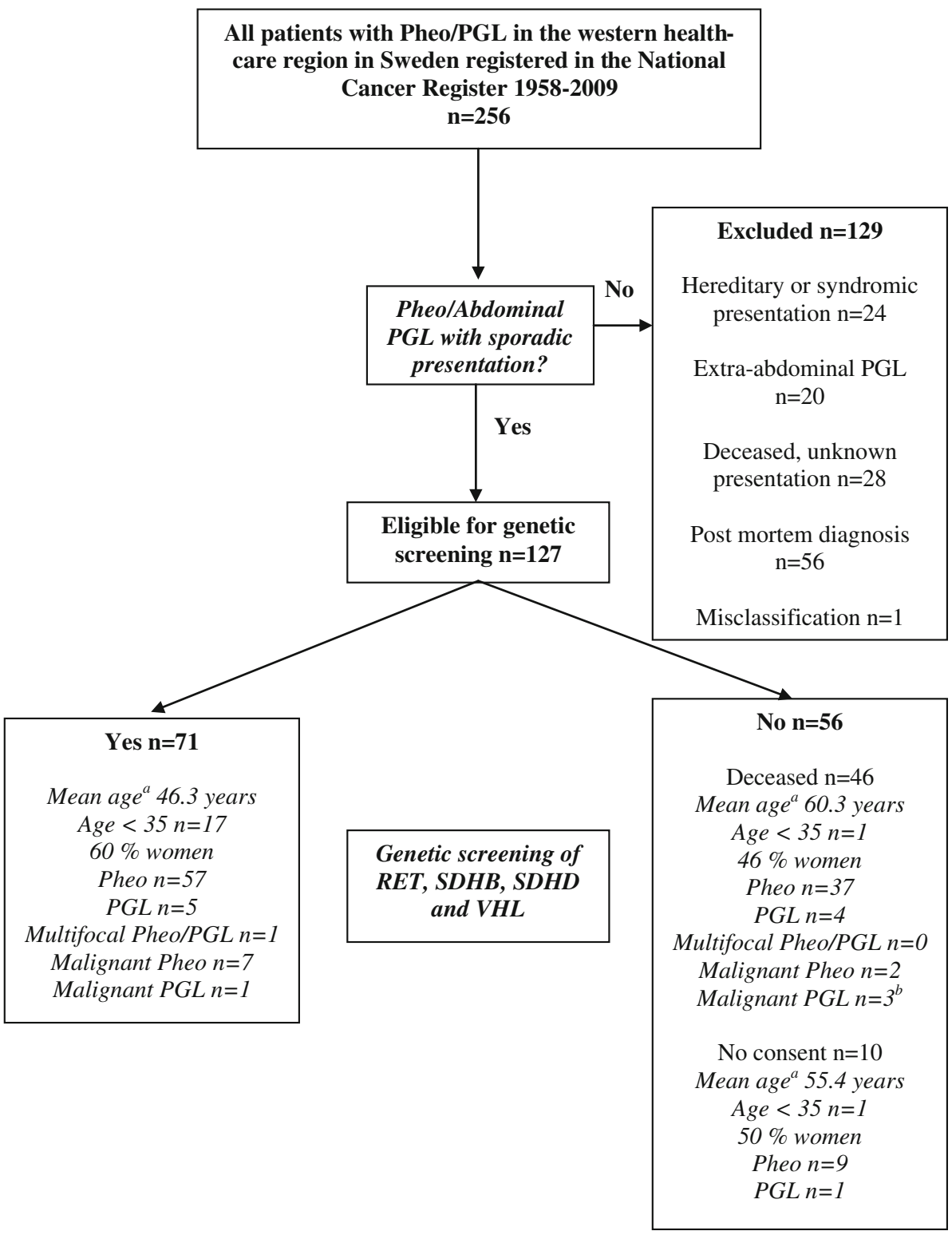


Table 1 Characteristics of the detected patients with germline mutations and sporadic presentation of pheochromocytoma or abdominal paraganglioma

\begin{tabular}{|c|c|c|c|c|c|c|c|c|}
\hline \multirow{2}{*}{$\begin{array}{l}\text { Age }(\text { years })^{\mathrm{a}} \text {, } \\
\text { sex }\end{array}$} & \multirow[t]{2}{*}{ Diagnosis } & \multirow{2}{*}{$\begin{array}{l}\text { Malignant/ } \\
\text { bilat/multif }^{\mathrm{b}}\end{array}$} & \multirow[t]{2}{*}{ Secr } & \multicolumn{2}{|l|}{ Mutation } & \multirow[t]{2}{*}{ LOVD ID $^{c}$} & \multirow[t]{2}{*}{ FU (years) } & \multirow[t]{2}{*}{ Status } \\
\hline & & & & c.DNA & Protein & & & \\
\hline $25, \mathrm{~F}$ & Pheo & No & $\mathrm{E}$ & $S D H B$ c. $716 \mathrm{C}>\mathrm{G}$ & p.Ser239Cys & Submitted & 26.0 & NED \\
\hline 49, M & Pheo & No & $\mathrm{NE}$ & $S D H B$ c. $725 \mathrm{G}>\mathrm{A}$ & p.Arg242His & SDHB_00004 & 15.7 & NED \\
\hline $15, \mathrm{~F}$ & PGL & No & NE & $S D H B$ c.IVS $4+1 \mathrm{G}>\mathrm{A}$ & Splice-site & SDHB_00047 & 28.1 & NED \\
\hline $27, \mathrm{~F}$ & Pheo & No & $\mathrm{E}$ & $R E T$ c $.1826 \mathrm{G}>\mathrm{A}$ & p.Cys609Tyr & - & 13 & NED \\
\hline
\end{tabular}

Pheo pheochromocytoma, $P G L$ paraganglioma, Secr secretory pattern, $E$ epinephrine, $N E$ norepinephrine, $F U$ follow-up, $N E D$ no evidence of disease

a Age at diagnosis

b Malignant (metastases at presentation or during follow-up), bilateral or multifocal tumor

${ }^{c}$ Leiden Open Variation Database (http://chromium.liacs.nl/lovd_sdh/home.php?select_db=SDHB)

registry. Information on presentation was missing in 28 deceased patients (Pheo $n=6$, PGL $n=10$, malignant Pheo $n=9$, malignant PGL $n=3$; mean age 59.2 years, mean time from diagnosis to death 7.6 years). Fifty-six patients were diagnosed postmortem (Pheo $n=39$, PGL $n=5$, malignant Pheo $n=12$ ).

Of the 127 patients with sporadic presentation, 81 were alive and were invited to the study, and $71(88 \%)$ gave their consent and underwent testing. Forty-six of the 127 patients were dead; the mean survival time from diagnosis was 12 years. Patient characteristics are shown in Fig. 1.

Sequencing revealed one case with a missense mutation in the RET-gene, two cases with missense, and one case with a splice-site mutation in $S D H B$ (Table 1). Six patients had single nucleotide polymorphisms in $S D H B$ : c.18A $>$ C (p.Ala6Ala) $n=4$; c.24C $>$ T (p.Ser8Ser) $n=1$; c.487T $>C$ (p.Ser163Pro) $n=1$, Leiden Open Variants Database (LOVD) ID SDHB_00008,SDHB_00011, and SDHB_00038, respectively (http://chromium.liacs.nl/lovd_sdh/home.php?select_db=SDHB) [15]. No mutations were found in $S D H D$ or $V H L$. No deletions or rearrangements were found by MLPA analysis.

The patient with a previously unrecognized RET p.Cys609Tyr mutation underwent a prophylactic thyroidectomy at age 40 years, 13 years after surgery for Pheo. At that time, she had no signs of recurrence of Pheo. On histopathological analysis, no $\mathrm{C}$ cell hyperplasia or medullary thyroid carcinoma was found. All three patients with $S D H B$ mutations are alive without evidence of tumor recurrence or malignant development at 15.7, 26.0, and 28.1 years of follow-up, respectively (Table 1 ).

\section{Discussion}

In this population-based study of patients with Pheo or abdominal PGL with sporadic presentation registered in the National Cancer Registry for Western Sweden 1958-2009, the prevalence of germline mutations was $5.6 \%$. Mutations were only seen in $R E T$ and $S D H B$. Notably, in the patients with $S D H B$ mutations no evidence of malignancy was observed during a mean follow up of 23.3 years.

Differences in the reported frequency of germline mutations may reflect genetic differences in different populations/geographical areas and/or differences in patient selection. In the present study, $82 \%$ had isolated apparently sporadic single Pheo. Cascon et al. [10] reported $9 \%$ germline mutations in patients with a single Pheo/PGL and sporadic presentation, and only $2.3 \%$ in patients with a single apparently sporadic Pheo. In the present study, the susceptibility genes $R E T, S D H B, S D H D$, and $V H L$ were sequenced, and deletions were excluded in $S D H B, S D H C$, and $S D H D$-genes and VHL. The mutation detection methods used in this study are well-established techniques and have been used by other investigators in this context [9]. Whereas Sanger sequencing shows a $>99.6 \%$ sensitivity for unidirectional analysis of heterozygous base substitutions [16], the MLPA technique is reported to show a sensitivity of approximately $92 \%$ [17]. The genetic testing methodology used is therefore not likely to explain the low prevalence of mutations found in this study. SDHC-mutation in apparently sporadic abdominal PGL seems rare [18].

Considering established risk factors for hereditary disease [7], tested patients were more likely to have germline mutations than patients who did not consent to the study and patients who had died before the study started, because tested patients were younger and more frequently had malignant disease. Still, a significant number of patients in the total cohort were deceased and a higher mutation frequency among these cannot be excluded. No systematic screening of relatives of living or deceased patients has yet been performed. However, a 39-year-old son of one deceased man with a malignant PGL has been diagnosed with an $S D H B$ mutation (418 G>T, p.Val140Phe, LOVD 
ID SDHB_00095). He has no evidence of disease. The mutation status of the father is unknown (Fig. 1).

Hereditary tumors occur at a younger age than sporadic tumors. Age $<35$ years at presentation is a risk factor for hereditary disease [7]. In this study, tested patients aged $<35$ years at presentation had a mutation frequency of $17.6 \%$, compared to $1.9 \%$ in patients aged $>35$ years (Fig. 1; Table 1). It should be noted that three of four patients with germline mutations in the present study were younger than 30 years at presentation. In series of patients $<20$ years, one-third may be mutation carriers [9, 19].

$S D H B$ carriers develop a malignant phenotype in $34.3-37.5 \%$ of the cases $[20,21]$. In the present study, no patient with $S D H B$ mutation had developed malignancy at presentation or during long-time follow-up (mean 23.3 years). Hypermethylation of the $\mathrm{P} 16^{\mathrm{INK} 4 \mathrm{~A}}$ promotor has been associated with malignant phenotype in $S D H B$ carriers [22], indicating stepwise genetic changes during the malignant transformation. To better tailor the follow-up of $S D H B$ carriers, more information is needed on the impact of specific mutations and also how epigenetic alterations influence the phenotype.

Genetic analyses are time-consuming and costly. To minimize the number of analyses, most authors suggest a stepwise testing based on clinical data [8-10]. To speed up the process and cut costs, immunohistochemical analysis of SDHB protein expression in tumor tissue [23] has been used as a highly sensitive and specific screening tool to discriminate $S D H$-related from non-SDH-related tumours. Denaturing high performance liquid chromatography also has been used as a fast and relatively inexpensive screening method [24].

In this registry-based study, we found a frequency of germline mutations in patients with apparently sporadic pheochromocytoma and abdominal paraganglioma in Western Sweden of $5.6 \%$. Differences in reported frequencies of germline mutations in patients with clinically sporadic Pheo/PGL may reflect geographical differences or patient selection.

Acknowledgments The authors thank Associate Professor Fredrik Enlund, PhD, at the Department of Pathology, Sahlgrenska University Hospital, Gothenburg, Sweden, for expert assistance with the RETanalyses. This study was supported by the Region of Western Sweden, the Swedish Cancer Society, the Swedish Research Council, the Göteborg Medical Society, and the Assar Gabrielsson Foundation.

Conflict of interest The authors declare that they have no conflicts of interest.

Open Access This article is distributed under the terms of the Creative Commons Attribution Noncommercial License which permits any noncommercial use, distribution, and reproduction in any medium, provided the original author(s) and source are credited.

\section{References}

1. Ladroue C, Carcenac R, Leporrier M et al (2008) PHD2 mutation and congenital erythrocytosis with paraganglioma. N Engl J Med 359:2685-2692

2. Burnichon N, Briere JJ, Libe R et al (2010) SDHA is a tumor suppressor gene causing paraganglioma. Hum Mol Genet 19: 3011-3020

3. Qin Y, Yao L, King EE et al (2010) Germline mutations in TMEM127 confer susceptibility to pheochromocytoma. Nat Genet 42:229-233

4. Schlisio S, Kenchappa RS, Vredeveld LC et al (2008) The kinesin KIF1Bbeta acts downstream from EglN3 to induce apoptosis and is a potential 1p36 tumor suppressor. Genes Dev 22:884-893

5. Comino-Mendez I, Gracia-Aznarez FJ, Schiavi F et al (2011) Exome sequencing identifies MAX mutations as a cause of hereditary pheochromocytoma. Nat Genet 43:663-667

6. Neumann HP, Bausch B, McWhinney SR et al (2002) Germline mutations in nonsyndromic pheochromocytoma. N Engl J Med 346:1459-1466

7. Bornstein SR, Gimenez-Roqueplo AP (2006) Genetic testing in pheochromocytoma: increasing importance for clinical decision making. Ann N Y Acad Sci 1073:94-103

8. Burnichon N, Rohmer V, Amar L et al (2009) The succinate dehydrogenase genetic testing in a large prospective series of patients with paragangliomas. J Clin Endocrinol Metab 94:2817-2827

9. Mannelli M, Castellano M, Schiavi F et al (2009) Clinically guided genetic screening in a large cohort of Italian patients with pheochromocytomas and/or functional or nonfunctional paragangliomas. J Clin Endocrinol Metab 94:1541-1547

10. Cascon A, Pita G, Burnichon N et al (2009) Genetics of pheochromocytoma and paraganglioma in Spanish patients. J Clin Endocrinol Metab 94:1701-1705

11. Amar L, Bertherat J, Baudin E et al (2005) Genetic testing in pheochromocytoma or functional paraganglioma. J Clin Oncol 23:8812-8818

12. Korpershoek E, Van Nederveen FH, Dannenberg H et al (2006) Genetic analyses of apparently sporadic pheochromocytomas: the Rotterdam experience. Ann N Y Acad Sci 1073:138-148

13. Barlow L, Westergren K, Holmberg L et al (2009) The completeness of the Swedish Cancer Register: a sample survey for year 1998. Acta Oncol 48:27-33

14. Thompson LDR, Young WFJ, Kawashima A et al (2004) Malignant adrenal phaeochromocytoma. In: DeLellis RA, Lloyd RV, Heitz PU et al (eds) Pathology and genetics of tumours of endocrine organs. IARC Press, Lyon

15. Bayley JP, Devilee P, Taschner PE (2005) The SDH mutation database: an online resource for succinate dehydrogenase sequence variants involved in pheochromocytoma, paraganglioma and mitochondrial complex II deficiency. BMC Med Genet 6:39

16. Ellard S, Shields B, Tysoe C et al (2009) Semi-automated unidirectional sequence analysis for mutation detection in a clinical diagnostic setting. Genet Test Mol Biomark 13:381-386

17. Farshid G, Cheetham G, Davies R et al (2011) Validation of the multiplex ligation-dependent probe amplification (MLPA) technique for the determination of HER2 gene amplification in breast cancer. Diagn Mol Pathol 20:11-17

18. Mannelli M, Ercolino T, Giache V et al (2007) Genetic screening for pheochromocytoma: Should SDHC gene analysis be included? J Med Genet 44:586-587

19. Barontini M, Levin G, Sanso G (2006) Characteristics of pheochromocytoma in a 4- to 20-year-old population. Ann N Y Acad Sci 1073:30-37 
20. Benn DE, Gimenez-Roqueplo AP, Reilly JR et al (2006) Clinical presentation and penetrance of pheochromocytoma/paraganglioma syndromes. J Clin Endocrinol Metab 91:827-836

21. Neumann HP, Pawlu C, Peczkowska M et al (2004) Distinct clinical features of paraganglioma syndromes associated with SDHB and SDHD gene mutations. JAMA 292:943-951

22. Kiss NB, Geli J, Lundberg F et al (2008) Methylation of the p16INK4A promoter is associated with malignant behavior in abdominal extra-adrenal paragangliomas but not pheochromocytomas. Endocr Relat Cancer 15:609-621
23. van Nederveen FH, Gaal J, Favier J et al (2009) An immunohistochemical procedure to detect patients with paraganglioma and phaeochromocytoma with germline SDHB, SDHC, or SDHD gene mutations: a retrospective and prospective analysis. Lancet Oncol 10:764-771

24. Meyer-Rochow GY, Smith JM, Richardson AL et al (2009) Denaturing high-performance liquid chromatography detection of SDHB, SDHD, and VHL germline mutations in pheochromocytoma. J Surg Res 157:55-62 